PROCEEDINGS OF THE

AMERICAN MATHEMATICAL SOCIETY

Volume 138, Number 6, June 2010, Pages 1929-1939

S 0002-9939(10)10256-1

Article electronically published on January 22, 2010

\title{
HOPF CYCLIC COHOMOLOGY AND BIDERIVATIONS
}

\author{
ABHISHEK BANERJEE
}

(Communicated by Varghese Mathai)

\begin{abstract}
Hopf cyclic cohomology $H C_{(\delta, \sigma)}^{*}(\mathcal{H})$ for a Hopf algebra $\mathcal{H}$ with respect to a modular pair in involution $(\delta, \sigma)$ was introduced by Connes and Moscovici. By a biderivation $D$ on a Hopf algebra $\mathcal{H}$ we shall mean a linear map that satisfies the axioms for both a derivation and a coderivation on $\mathcal{H}$. Given a biderivation $D$ on a Hopf algebra, we define, under certain conditions, a map $L_{D}: H C_{(\delta, \sigma)}^{*}(\mathcal{H}) \longrightarrow H C_{(\delta, \sigma)}^{*}(\mathcal{H})$. We give examples of such maps for the quantized universal enveloping algebra $\mathcal{U}_{h}(\mathfrak{g})$ of a simple Lie algebra $\mathfrak{g}$. When $\mathcal{H}$ is irreducible, cocommutative and equipped with a character $\delta$ such that $(\delta, 1)$ is a modular pair in involution, we define "inner biderivations" and use these to produce a left $\mathcal{H}$-module structure on $H C_{(\delta, 1)}^{*}(\mathcal{H})$. Finally, we show that every morphism $L_{D}: H C_{(\delta, 1)}^{*}(\mathcal{H}) \longrightarrow H C_{(\delta, 1)}^{*}(\mathcal{H})$ induced by a biderivation $D$ on such a Hopf algebra $\mathcal{H}$ can be realized as a morphism induced by an inner biderivation by embedding $\mathcal{H}$ into a larger Hopf algebra $\mathcal{H}[D]$.
\end{abstract}

\section{INTRODUCTION}

Cyclic cohomology for Hopf algebras was introduced, in the context of transverse index theory of foliations, by Connes and Moscovici in 3, 4]. For a Hopf algebra $\mathcal{H}$ over $\mathbb{C}$ equipped with a "modular character" $\delta: \mathcal{H} \longrightarrow \mathbb{C}$ satisfying certain conditions, the authors of [3] construct a cocyclic module $\mathcal{H}_{\delta}^{\sharp}=\left\{\mathcal{H}^{\otimes n}\right\}_{n \geq 1}$. More generally, one can define a cocyclic module $\mathcal{H}_{(\delta, \sigma)}^{\sharp}$ corresponding to a Hopf algebra $\mathcal{H}$ equipped with a modular pair in involution $(\delta, \sigma)$ consisting of a character $\delta$ : $\mathcal{H} \longrightarrow \mathbb{C}$ and a "grouplike element" $\sigma \in \mathcal{H}$ satisfying certain conditions (see [3]). The Hopf cyclic cohomology $H C_{(\delta, \sigma)}^{*}(\mathcal{H})$ of $\mathcal{H}$ with respect to the pair $(\delta, \sigma)$ is defined to be the cohomology of this cocyclic module $\mathcal{H}_{(\delta, \sigma)}^{\sharp}$. This latter theory has been subsumed within the theory of stable anti-Yetter-Drinfeld modules developed by Hajac, Khalkhali, Rangipour and Sommerhäuser (see [6], 7]).

Given an algebra $A$ over $\mathbb{C}$, a derivation $D$ on $A$ induces a map $L_{D}: H C_{*}(A) \longrightarrow$ $H C_{*}(A)$ on the cyclic homology $H C_{*}(A)$ of $A$ (see [8, $\left.\S 4.1\right]$ ). The map $L_{D}$ plays the role of a Lie derivative in noncommutative geometry. In the present paper, we are concerned with understanding the maps induced on the Hopf cyclic cohomology by a "biderivation" of a Hopf algebra $\mathcal{H}$ under certain extra conditions. By a biderivation $D$ on $\mathcal{H}$ we will mean a linear map $D: \mathcal{H} \longrightarrow \mathcal{H}$ that satisfies both

Received by the editors April 9, 2009, and, in revised form, September 13, 2009.

2010 Mathematics Subject Classification. Primary 16W25, 16T05, 57T05.

Key words and phrases. Hopf cyclic cohomology, derivations, coderivations.

(C)2010 American Mathematical Society Reverts to public domain 28 years from publication 
the axioms for being a derivation on the algebra underlying $\mathcal{H}$ and the axioms for being a coderivation on the coalgebra underlying $\mathcal{H}$. In this respect, our first result is the following:

Proposition 0.1. Let $\mathcal{H}$ be a Hopf algebra over $\mathbb{C}$ equipped with a modular pair in involution $(\delta, \sigma)$. Then, if $D$ is a biderivation on $\mathcal{H}$ that satisfies (1.11), $D$ induces a morphism

$$
L_{D}: H C_{(\delta, \sigma)}^{*}(\mathcal{H}) \longrightarrow H C_{(\delta, \sigma)}^{*}(\mathcal{H})
$$

of Hopf cyclic cohomologies of $\mathcal{H}$.

Thereafter, we demonstrate biderivations $D$ that induce maps $L_{D}$ as in (0.1) on the Hopf cyclic cohomology of the quantized universal enveloping algebra $\mathcal{U}_{h}(\mathfrak{g})$ of a finite dimensional, complex, simple Lie algebra $\mathfrak{g}$. This example is constructed in Proposition 1.7.

Then we consider the case of a Hopf algebra $\mathcal{H}$ over $\mathbb{C}$ that is irreducible and cocommutative, i.e., the universal enveloping algebra of a Lie algebra $\mathfrak{g}$. In this case, the elements of $\mathcal{H}$ may be used to induce inner biderivations (see Definition 2.2) on $\mathcal{H}$ and hence we obtain the following result:

Proposition 0.2. Let $\mathcal{H}$ be a Hopf algebra over $\mathbb{C}$ equipped with a character $\delta$ : $\mathcal{H} \longrightarrow \mathbb{C}$ such that $(\delta, 1)$ is a modular pair in involution. Suppose, moreover, that $\mathcal{H}$ is irreducible and cocommutative. Then, the Hopf cyclic cohomology $\mathrm{HC}_{(\delta, 1)}^{*}(\mathcal{H})$ carries the structure of a left $\mathcal{H}$-module.

When the Hopf algebra $\mathcal{H}$ is equipped with a character $\delta: \mathcal{H} \longrightarrow \mathbb{C}$ such that $(\delta, 1)$ is a modular pair in involution, we will denote the corresponding Hopf cyclic cohomology $H C_{(\delta, 1)}^{*}(\mathcal{H})$ simply by $H C_{\delta}^{*}(\mathcal{H})$. Finally, suppose that we have an irreducible and cocommutative Hopf algebra $\mathcal{H}$ over $\mathbb{C}$ with a biderivation $D$ acting on it and that $D$ induces an operator $L_{D}: H C_{\delta}^{*}(\mathcal{H}) \longrightarrow H C_{\delta}^{*}(\mathcal{H})$ on its Hopf cyclic cohomology. We want to show that the operator $L_{D}$ can be interpreted as a map induced by an inner biderivation. Therefore, we construct a Hopf algebra $\mathcal{H}[D]$ into which $\mathcal{H}$ embeds, along with a character $\delta_{D}: \mathcal{H}[D] \longrightarrow \mathbb{C}$ extending $\delta: \mathcal{H} \longrightarrow \mathbb{C}$, and we have the following result:

Proposition 0.3. Let $\mathcal{H}$ be a Hopf algebra over $\mathbb{C}$ equipped with a character $\delta$ : $\mathcal{H} \longrightarrow \mathbb{C}$ such that $(\delta, 1)$ is a modular pair in involution. Suppose, moreover, that $\mathcal{H}$ is irreducible and cocommutative. Let $D$ be a biderivation on $\mathcal{H}$ that satisfies (1.11). Then there exists a Hopf algebra $\mathcal{H}[D]$ along with a character $\delta_{D}: \mathcal{H} \longrightarrow \mathbb{C}$ extending $\delta$ and an embedding $i: \mathcal{H} \longrightarrow \mathcal{H}[D]$ such that there exists an inner biderivation $(D): \mathcal{H}[D] \longrightarrow \mathcal{H}[D]$ on $\mathcal{H}[D]$ inducing a commutative diagram

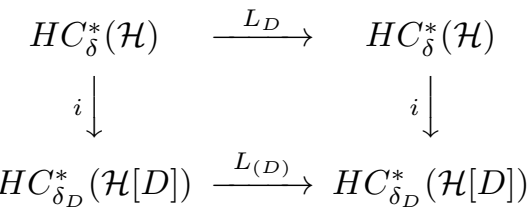

The organisation of this paper is as follows: the framework for Hopf cyclic cohomology is briefly recalled in Section 1 . Thereafter, we construct the morphism $L_{D}$ on Hopf cyclic cohomology due to a biderivation on a Hopf algebra described in Proposition 0.1 and give examples for quantized universal enveloping algebras $\mathcal{U}_{h}(\mathfrak{g})$. In Section 2, we introduce our concept of inner biderivations and exhibit 
the left $\mathcal{H}$-module structure on the Hopf cyclic cohomology mentioned in Proposition 0.2 above. Finally, in Section 3, we construct the Hopf algebra $\mathcal{H}[D]$ and prove Proposition 0.3 .

\section{THE OPERATOR INDUCED BY A BIDERIVATION ON HOPF CYCLIC COHOMOLOGY}

Let $(\mathcal{H}, m, \Delta, u, \epsilon, S)$ be a Hopf algebra over $\mathbb{C}$ with unit map $u: \mathbb{C} \rightarrow \mathcal{H}$, counit map $\epsilon: \mathcal{H} \rightarrow \mathbb{C}$, algebra product $m: \mathcal{H} \otimes \mathcal{H} \longrightarrow \mathcal{H}$, coalgebra coproduct $\Delta: \mathcal{H} \longrightarrow \mathcal{H} \otimes \mathcal{H}$ and antipode $S: \mathcal{H} \rightarrow H$.

Throughout, we shall use the standard Sweedler notation; i.e. we write

$$
\Delta(h)=\sum h_{(1)} \otimes h_{(2)} \quad \forall h \in \mathcal{H}
$$

We fix a character $\delta: \mathcal{H} \longrightarrow \mathbb{C}$ of $\mathcal{H}$ (which we shall refer to as the modular character) and consider the twisted antipode $\tilde{S}$ defined as

$$
\tilde{S}(h)=(\delta * S)(h)=\sum \delta\left(h_{(1)}\right) S\left(h_{(2)}\right) .
$$

Consider a "grouplike element" $\sigma$ of $\mathcal{H}$ (i.e., an element $\sigma \in \mathcal{H}$ such that $\Delta(\sigma)=$ $\sigma \otimes \sigma)$. Suppose that the character $\delta: \mathcal{H} \longrightarrow \mathbb{C}$ satisfies

$$
\delta(\sigma)=1 \text {. }
$$

Then, the pair $(\delta, \sigma)$ is said to be a modular pair. Further, if the pair $(\delta, \sigma)$ is such that

$$
\left(\sigma^{-1} \tilde{S}\right)^{2}=1
$$

the pair $(\delta, \sigma)$ is said to be a modular pair in involution. It follows from these definitions (see [3]) that

$$
\begin{aligned}
& \tilde{S}\left(h^{1} h^{2}\right)=\tilde{S}\left(h^{2}\right) \tilde{S}\left(h^{1}\right) \\
& \tilde{S}(1)=1 \\
& \Delta \tilde{S}(h)=\sum S\left(h_{(2)}\right) \otimes \tilde{S}\left(h_{(1)}\right) \\
& \epsilon \circ \tilde{S}=\delta .
\end{aligned}
$$

We now briefly recall the definition of Hopf cyclic cohomology for a Hopf algebra endowed with a modular pair in involution due to Connes and Moscovici [3]. Consider the cosimplicial module $\left\{\mathcal{H}^{\otimes n}\right\}_{n \geq 1}$ with face operators $\delta_{i}: \mathcal{H}^{\otimes n-1} \rightarrow \mathcal{H}^{\otimes n}$,

$$
\begin{aligned}
& \delta_{0}\left(h^{1} \otimes \ldots \otimes h^{n-1}\right)=1 \otimes h^{1} \otimes \ldots \otimes h^{n-1} \\
& \delta_{i}\left(h^{1} \otimes \ldots \otimes h^{n-1}\right)=h^{1} \otimes \ldots \otimes \Delta h^{i} \otimes \ldots \otimes h^{n-1}, 1 \leq i \leq n-1 \\
& \delta_{n}\left(h^{1} \otimes \ldots \otimes h^{n-1}\right)=h^{1} \otimes \ldots \otimes h^{n-1} \otimes \sigma
\end{aligned}
$$

and degeneracy operators $\sigma_{i}: \mathcal{H}^{\otimes n+1} \rightarrow \mathcal{H}^{\otimes n}$

$$
\sigma_{i}\left(h^{1} \otimes \ldots \otimes h^{n+1}\right)=h^{1} \otimes \ldots \otimes \epsilon\left(h^{i+1}\right) \otimes \ldots \otimes h^{n+1}, \quad 0 \leq i \leq n .
$$

Furthermore, define the cyclic operator

$$
\tau_{n}\left(h^{1} \otimes \ldots \otimes h^{n}\right)=\left(\Delta^{n-1} \tilde{S}\left(h^{1}\right)\right) \cdot h^{2} \otimes \ldots \otimes h^{n} \otimes \sigma .
$$

If the pair $(\delta, \sigma)$ is a modular pair in involution, i.e. $\left(\sigma^{-1} \tilde{S}\right)^{2}=I$, [3, Theorem 1] shows that the operators $\tau_{n}$ on the cosimplicial module $\left\{\mathcal{H}^{\otimes n}\right\}_{n \geq 1}$ make it a cocyclic module, which we denote by $\mathcal{H}_{(\delta, \sigma)}^{\sharp}$. 
Definition 1.1. Let $\mathcal{H}$ be a Hopf algebra over $\mathbb{C}$ equipped with a modular pair in involution $(\delta, \sigma)$. The cohomology of the cocyclic module $\mathcal{H}_{(\delta, \sigma)}^{\sharp}$ is the Hopf cyclic cohomology $H C_{(\delta, \sigma)}^{*}(\mathcal{H})$ of $\mathcal{H}$ relative to the modular pair $(\delta, \sigma)$.

In particular, if the Hopf algebra $\mathcal{H}$ is equipped with a character $\delta: \mathcal{H} \longrightarrow \mathbb{C}$ such that $(\delta, 1)$ defines a modular pair in involution, we shall denote the Hopf cyclic cohomology of $\mathcal{H}$ relative to $(\delta, 1)$ simply by $H C_{\delta}^{*}(\mathcal{H})$ and the corresponding cocyclic module by $\mathcal{H}_{\delta}^{\sharp}$.

Henceforth, we shall always assume that our Hopf algebras are defined over $\mathbb{C}$ and carry a modular pair in involution $(\delta, \sigma)$. We are interested in the morphisms induced on the Hopf cyclic cohomology of a Hopf algebra $\mathcal{H}$ by maps that we shall refer to as "biderivations".

A biderivation on $\mathcal{H}$ shall be a linear map $D: \mathcal{H} \longrightarrow \mathcal{H}$ that satisfies the axioms both for being a derivation on the underlying algebra of $\mathcal{H}$ and for being a coderivation on the underlying coalgebra of $\mathcal{H}$. Therefore, suppose that we have a linear map $D: \mathcal{H} \rightarrow \mathcal{H}$ satisfying the following conditions:

(a) $D$ is a coderivation for the underlying coalgebra of $\mathcal{H}$; i.e. given $h \in \mathcal{H}$, we have

$$
\Delta(D(h))=(D \otimes 1+1 \otimes D) \Delta(h) .
$$

(b) $D$ is a derivation for the underlying algebra of $\mathcal{H}$; i.e. given $h^{1}, h^{2} \in \mathcal{H}$,

$$
D\left(h^{1} h^{2}\right)=D\left(h^{1}\right) h^{2}+h^{1} D\left(h^{2}\right) .
$$

(c) The following relations hold:

$$
\epsilon \circ D=0 \quad D \circ u=0 \quad D S=S D \quad \delta \circ D=0 \quad D(\sigma)=0 .
$$

If we extend the operator $D$ to any $\mathcal{H}^{\otimes n}, n \geq 1$, by setting

$$
D\left(h^{1} \otimes \ldots \otimes h^{n}\right)=\left(D\left(h^{1}\right) \otimes \ldots \otimes h^{n}\right)+\ldots+\left(h^{1} \otimes \ldots \otimes D\left(h^{n}\right)\right)
$$

for any $h^{1}, h^{2}, \ldots, h^{n} \in \mathcal{H}$, we can express condition (a) more compactly as

$$
\Delta \circ D(h)=D \circ \Delta(h) \quad \forall h \in \mathcal{H} .
$$

A linear map $D: \mathcal{H} \longrightarrow \mathcal{H}$ that satisfies conditions (a) and (b) above will be referred to as a biderivation. We will refer to the module of all biderivations on $\mathcal{H}$ as $\operatorname{Bider}(\mathcal{H})$. The submodule of all biderivations that also satisfy (c) will be referred to as $\operatorname{Bider}_{0}^{(\delta, \sigma)}(\mathcal{H})$. The pair $(\delta, \sigma)$ will usually be clear from the context and hence we will denote this subset simply by $\operatorname{Bider}_{0}(\mathcal{H})$.

Lemma 1.2. Let $\mathcal{H}$ be a Hopf algebra and let $D: \mathcal{H} \longrightarrow \mathcal{H}$ be a biderivation on $\mathcal{H}$ as described above. Then, if additionally $D \in \operatorname{Bider}_{0}(\mathcal{H})$ :

(a) The map $D$ commutes with the face maps $\delta_{i}$ and the degeneracy operators $\sigma_{j}$ of the cocyclic module $\mathcal{H}_{(\delta, \sigma)}^{\sharp}$.

(b) $D$ commutes with the twisted antipode; i.e. $D \circ \tilde{S}(y)=\tilde{S} \circ D(y)$ for all $y \in \mathcal{H}$. 
Proof. (a) We have maps $\delta_{i}: \mathcal{H}^{\otimes n-1} \rightarrow \mathcal{H}^{\otimes n}, 0 \leq i \leq n$. The result is obvious for $i=0$ and for $i=n$ (since $D(\sigma)=0)$. For $1 \leq i \leq n-1$, we have

$(1.14)$

$$
\begin{aligned}
& D\left(\delta_{i}\left(h^{1} \otimes \ldots \otimes h^{n-1}\right)\right)= D\left(\sum h^{1} \otimes \ldots \otimes h_{(1)}^{i} \otimes h_{(2)}^{i} \otimes \ldots \otimes h^{n-1}\right) \\
&= \sum_{j=1}^{i-1}\left(h^{1} \otimes \ldots \otimes h^{j-1} \otimes D\left(h^{j}\right) \otimes h^{j+1} \otimes \ldots\right. \\
&\left.+\sum h^{1} \otimes \ldots \otimes h^{i-1} \otimes h_{(1)}^{i} \otimes h_{(2)}^{i} \otimes \ldots \otimes h^{n-1}\right) \\
&+\sum^{i-1} h^{1} \otimes \ldots \otimes h^{i-1} \otimes h_{(1)}^{i} \otimes D \otimes h_{(2)}^{i} \otimes \ldots \otimes h^{n-1} \\
&+\sum_{j=i+1}^{n-1}\left(h^{1} \otimes \ldots \otimes h_{(1)}^{i} \otimes h_{(2)}^{i} \otimes h^{j-1}\right. \\
&= \delta_{i}\left(D \left(h^{1} \otimes \ldots \otimes h^{n-1}\right.\right. \\
&
\end{aligned}
$$

For the degeneracy operators, we have

$(1.15)$

$$
\begin{aligned}
& D\left(\sigma_{i}\left(h^{1} \otimes \ldots \otimes h^{n+1}\right)\right)=D\left(h^{1} \otimes \ldots \otimes \epsilon\left(h^{i+1}\right) \otimes \ldots \otimes h^{n+1}\right) \\
& =\epsilon\left(h^{i+1}\right) \sum_{j=1}^{i}\left(h^{1} \otimes \ldots \otimes h^{j-1} \otimes D\left(h^{j}\right) \otimes h^{j+1} \otimes \ldots\right. \\
& \left.\otimes h^{i} \otimes h^{i+2} \otimes \ldots \otimes h^{n+1}\right) \\
& +\epsilon\left(h^{i+1}\right) \sum_{j=i+2}^{n+1}\left(h^{1} \otimes \ldots \otimes h^{i} \otimes h^{i+2} \otimes \ldots \otimes h^{j-1}\right. \\
& =\sigma_{i}\left(D\left(h^{1} \otimes \ldots \otimes h^{n+1}\right) \quad(\text { since } \epsilon \circ D=0) .\right.
\end{aligned}
$$

(b) Let $y \in \mathcal{H}$. Then

$$
\begin{aligned}
\tilde{S}(D(y)) & =(\delta * S)(D(y)) \\
& =\sum \delta\left(D\left(y_{(1)}\right)\right) S\left(y_{(2)}\right)+\sum \delta\left(y_{(1)}\right) S\left(D y_{(2)}\right) \\
& =\sum \delta\left(y_{(1)}\right) D\left(S\left(y_{(2)}\right)\right) \quad(\text { since } \delta \circ D=0) \\
& =D\left(\sum \delta\left(y_{(1)}\right) S\left(y_{(2)}\right)\right)=D(\delta * S)(y)=D \circ \tilde{S}(y) .
\end{aligned}
$$

Lemma 1.3. Let $\mathcal{H}$ be a Hopf algebra and let $D: \mathcal{H} \longrightarrow \mathcal{H}$ be a biderivation on $\mathcal{H}$ as described above. Then, if additionally $D \in \operatorname{Bider}_{0}(\mathcal{H}), D$ commutes with the cyclic operators $\tau_{n}$ on the cocyclic module $\mathcal{H}_{(\delta, \sigma)}^{\sharp}=\left\{\mathcal{H}^{\otimes n}\right\}_{n \geq 1}$.

Proof. We note that the extension of the operator $D$ to $\mathcal{H}^{\otimes n}$ is also a derivation; i.e. given $h^{1} \otimes h^{2} \otimes \ldots \otimes h^{n}, k^{1} \otimes k^{2} \otimes \ldots \otimes k^{n} \in \mathcal{H}^{\otimes n}$, we have

$$
\begin{aligned}
D\left(\left(h^{1} \otimes h^{2} \otimes \ldots\right.\right. & \left.\left.\otimes h^{n}\right) \cdot\left(k^{1} \otimes k^{2} \otimes \ldots \otimes k^{n}\right)\right) \\
= & D\left(h^{1} \otimes h^{2} \otimes \ldots \otimes h^{n}\right) \cdot\left(k^{1} \otimes k^{2} \otimes \ldots \otimes k^{n}\right) \\
& +\left(h^{1} \otimes h^{2} \otimes \ldots \otimes h^{n}\right) \cdot D\left(k^{1} \otimes k^{2} \otimes \ldots \otimes k^{n}\right) .
\end{aligned}
$$

By definition,

$$
\tau_{n}\left(h^{1} \otimes \ldots \otimes h^{n}\right)=\left(\Delta^{n-1} \tilde{S}\left(h^{1}\right)\right) \cdot\left(h^{2} \otimes \ldots \otimes h^{n} \otimes \sigma\right) .
$$


We have noted before that $D \circ \Delta=\Delta \circ D$, and we have $D \circ \tilde{S}=\tilde{S} \circ D$ from Lemma 1.2 above. Hence,

$$
\begin{aligned}
D \tau_{n}\left(h^{1} \otimes \ldots \otimes h^{n}\right)= & D\left(\left(\Delta^{n-1} \tilde{S}\left(h^{1}\right)\right) \cdot\left(h^{2} \otimes \ldots \otimes h^{n} \otimes \sigma\right)\right) \\
= & D\left(\Delta^{n-1} \tilde{S}\left(h^{1}\right)\right) \cdot\left(h^{2} \otimes \ldots \otimes h^{n} \otimes \sigma\right) \\
& +\left(\Delta^{n-1} \tilde{S}\left(h^{1}\right)\right) \cdot D\left(h^{2} \otimes \ldots \otimes h^{n} \otimes \sigma\right) \\
= & \left(\Delta^{n-1} \tilde{S}\left(D h^{1}\right)\right) \cdot\left(h^{2} \otimes \ldots \otimes h^{n} \otimes \sigma\right) \\
& +\sum_{i=2}^{n}\left(\Delta^{n-1} \tilde{S}\left(h^{1}\right)\right) \cdot\left(h^{2} \otimes \ldots \otimes D\left(h^{i}\right) \otimes \ldots \otimes h^{n} \otimes \sigma\right) \\
= & \tau_{n}\left(D\left(h^{1}\right) \otimes h^{2} \otimes \ldots \otimes h^{n}\right) \\
& +\sum_{i=2}^{n} \tau_{n}\left(h^{1} \otimes \ldots \otimes h^{i-1} \otimes D\left(h^{i}\right) \otimes \ldots \otimes h^{n}\right) \\
= & \tau_{n}\left(D\left(h^{1} \otimes \ldots \otimes h^{n}\right)\right) .
\end{aligned}
$$

We are now ready to define the operator $L_{D}: H C_{(\delta, \sigma)}^{*}(\mathcal{H}) \longrightarrow H C_{(\delta, \sigma)}^{*}(\mathcal{H})$.

Proposition 1.4. Let $\mathcal{H}$ be a Hopf algebra equipped with a modular pair in involution $(\delta, \sigma)$ and let $D: \mathcal{H} \longrightarrow \mathcal{H}$ be a biderivation on $\mathcal{H}$ as described above. Then, if additionally $D \in$ Bider $_{0}(\mathcal{H})$, the map $D$ induces a morphism in Hopf cyclic cohomology:

$$
L_{D}: H C_{(\delta, \sigma)}^{*}(\mathcal{H}) \rightarrow H C_{(\delta, \sigma)}^{*}(\mathcal{H}) .
$$

Proof. This follows directly from the fact that the action of $D \in \operatorname{Bider}_{0}(\mathcal{H})$ commutes with the face maps, degeneracies and cyclic operators on the cocyclic module $\mathcal{H}_{(\delta, \sigma)}^{\sharp}$ defining Hopf cyclic cohomology.

We will now consider the commutator $\left[D_{1}, D_{2}\right]$ of two biderivations $D_{1}$ and $D_{2}$ on $\mathcal{H}$. These calculations will be useful in Section 2 when we define the left $\mathcal{H}$ module structure on $H C_{\delta}^{*}(\mathcal{H})$ for an irreducible, cocommutative Hopf algebra $\mathcal{H}$ over $\mathbb{C}$ equipped with a character $\delta: \mathcal{H} \longrightarrow \mathbb{C}$ such that $(\delta, 1)$ is a modular pair in involution.

Lemma 1.5. Let $\mathcal{H}$ be a Hopf algebra equipped with a modular pair in involution $(\delta, \sigma)$ and let $D_{1}, D_{2} \in \operatorname{Bider}_{0}(\mathcal{H})$. Then, the commutator $\left[D_{1}, D_{2}\right] \in \operatorname{Bider}_{0}(\mathcal{H})$.

Proof. Let $D_{1}, D_{2} \in \operatorname{Bider}_{0}(\mathcal{H})$. The commutator $\left[D_{1}, D_{2}\right]$ is a derivation for the algebra structure of $\mathcal{H}$. To verify that $\left[D_{1}, D_{2}\right]$ is a coderivation, we check, for $h \in \mathcal{H}$, that

$$
\begin{aligned}
\Delta\left(D_{1} D_{2}(h)\right)= & \left(D_{1} \otimes 1+1 \otimes D_{1}\right) \Delta\left(D_{2}(h)\right) \\
= & \left(D_{1} \otimes 1+1 \otimes D_{1}\right)\left(D_{2} \otimes 1+1 \otimes D_{2}\right)(\Delta(h)) \\
\Delta\left(D_{2} D_{1}(h)\right)= & \left(D_{2} \otimes 1+1 \otimes D_{2}\right)\left(D_{1} \otimes 1+1 \otimes D_{1}\right)(\Delta(h)) \\
\Rightarrow \Delta\left(\left[D_{1}, D_{2}\right](h)\right)= & \left(\left[D_{1}, D_{2}\right] \otimes 1+1 \otimes\left[D_{1}, D_{2}\right]\right)(\Delta(h)) .
\end{aligned}
$$

Since furthermore $D_{1}$ and $D_{2}$ lie in $\operatorname{Bider}_{0}(\mathcal{H})$, it follows that $\epsilon \circ\left[D_{1}, D_{2}\right]=0$, $\left[D_{1}, D_{2}\right] \circ u=0,\left[D_{1}, D_{2}\right] S=S\left[D_{1}, D_{2}\right],\left[D_{1}, D_{2}\right](\sigma)=0$ and $\delta \circ\left[D_{1}, D_{2}\right]=0$. Hence, $\left[D_{1}, D_{2}\right] \in \operatorname{Bider}_{0}(\mathcal{H})$. 
Proposition 1.6. Let $\mathcal{H}$ be a Hopf algebra equipped with a modular pair in involution $(\delta, \sigma)$ and let $D_{1}, D_{2} \in \operatorname{Bider}_{0}(\mathcal{H})$. Then,

$$
L_{\left[D_{1}, D_{2}\right]}=\left[L_{D_{1}}, L_{D_{2}}\right]: H C_{(\delta, \sigma)}^{*}(\mathcal{H}) \longrightarrow H C_{(\delta, \sigma)}^{*}(\mathcal{H}) .
$$

Proof. From Lemma 1.5, it follows that $\left[D_{1}, D_{2}\right] \in \operatorname{Bider}_{0}(\mathcal{H})$. Hence, the operator $L_{\left[D_{1}, D_{2}\right]}$ descends to the cohomology $H C_{(\delta, \sigma)}^{*}(\mathcal{H})$. It follows directly from the definitions that $L_{\left[D_{1}, D_{2}\right]}=\left[L_{D_{1}}, L_{D_{2}}\right]$.

We end this section by giving examples of such maps $L_{D}$ for the quantized universal enveloping algebra $\mathcal{U}_{h}(\mathfrak{g})$ of a finite dimensional, complex, simple Lie algebra $\mathfrak{g}$ of rank $N$. Let $\left\{\alpha_{1}, \ldots, \alpha_{N}\right\}$ be a system of simple roots and let (,) denote the canonical bilinear form on the root space obtained from the Killing form. Then, the Cartan matrix $A=\left(\left(a_{i j}\right)\right)$ of $\mathfrak{g}$ has the description

$$
a_{i j}=2 \frac{\left(\alpha_{i}, \alpha_{j}\right)}{\left(\alpha_{i}, \alpha_{i}\right)}, \quad i, j=1,2, \ldots, N
$$

and we set

$$
d_{i}=\frac{\left(\alpha_{i}, \alpha_{i}\right)}{2}, \quad i=1,2, \ldots, N .
$$

For any invertible $q \in \mathbb{C}[[h]]$ in the ring of formal power series, define the $q$ integers by

$$
[n]_{q}=\frac{q^{n}-q^{-n}}{q-q^{-1}}=q^{n-1}+q^{n-3}+\cdots+q^{-n+3}+q^{-n+1}
$$

for any integer $n$. Then, for any non-negative integer $k$, the $q$-binomial coefficients are defined by

$$
[k]_{q}=\prod_{i=1}^{k}[i]_{q} \quad\left(\begin{array}{l}
n \\
k
\end{array}\right)_{q}=\frac{[n]_{q} !}{[n-k]_{q} ![k]_{q} !} .
$$

The quantized universal enveloping algebra $\mathcal{U}_{h}(\mathfrak{g})$ can now be defined as an associative algebra over $\mathbb{C}[[h]]$, complete in $h$-adic topology, through the generators $\left\{H_{i}, X_{ \pm i}\right\}, i=1,2, \ldots, N$, and the relations (see [10, $\left.\S 7.1\right]$ )

$$
\begin{gathered}
{\left[H_{i}, H_{j}\right]=0 \quad\left[H_{i}, X_{ \pm j}\right]= \pm a_{i j} X_{ \pm j} \quad\left[X_{+i}, X_{-j}\right]=\delta_{i j} \frac{q_{i}^{H_{i}}-q_{i}^{-H_{i}}}{q_{i}-q_{i}^{-1}}} \\
\sum_{k=0}^{1-a_{i j}}(-1)^{k}\left(\begin{array}{c}
1-a_{i j} \\
k
\end{array}\right)_{q_{i}} X_{ \pm i}^{1-a_{i j}-k} X_{ \pm j} X_{ \pm i}^{k}=0 \quad \forall i \neq j .
\end{gathered}
$$

It can be shown that there is an isomorphism $\mathcal{U}_{h}(\mathfrak{g}) \cong \mathcal{U}(\mathfrak{g})[[h]]$ of $\mathbb{C}[[h]]$-modules (see [5]), where $\mathcal{U}(\mathfrak{g})$ is the universal enveloping algebra of $\mathfrak{g}$. We will treat $\mathcal{U}_{h}(\mathfrak{g})$ as a topological Hopf algebra, i.e., we will say that the coproduct $\Delta$ on $\mathcal{U}_{h}(\mathfrak{g})$ takes values in the completion $\mathcal{U}_{h}(\mathfrak{g}) \hat{\otimes}_{\mathcal{U}}(\mathfrak{g})$ of the tensor product $\mathcal{U}_{h}(\mathfrak{g}) \otimes_{\mathbb{C}[h]]} \mathcal{U}_{h}(\mathfrak{g})$ in $h$-adic topology. Then, the coproduct, the unit and the antipode are defined by

$$
\begin{array}{cc}
\Delta H_{i}=H_{i} \otimes 1+1 \otimes H_{i} & \Delta X_{ \pm i}=X_{ \pm i} \otimes q_{i}^{H_{i} / 2}+q_{i}^{H_{i} / 2} \otimes X_{ \pm i} \\
\epsilon\left(H_{i}\right)=\epsilon\left(X_{ \pm i}\right)=0 & S\left(H_{i}\right)=-H_{i} \quad S\left(X_{ \pm i}\right)=-q_{i}^{ \pm 1} X_{ \pm i} .
\end{array}
$$

Given a modular pair in involution $(\delta, \sigma)$ for the Hopf algebra $\mathcal{U}_{h}(\mathfrak{g})$, the definitions at the beginning of this section can be extended directly to the topological case to define the topological cocyclic module $\mathcal{U}_{h}(\mathfrak{g})_{(\delta, \sigma)}^{\sharp, t o p}=\left\{\mathcal{U}_{h}(\mathfrak{g})^{\otimes} \hat{\otimes}\right\}$. We will 
use $H C_{(\delta, \sigma)}^{*}\left(\mathcal{U}_{h}(\mathfrak{g})\right)$ to denote the cohomology of the cocyclic module $\mathcal{U}_{h}(\mathfrak{g})_{(\delta, \sigma)}^{\sharp, \text { top }}=$ $\left\{\mathcal{U}_{h}(\mathfrak{g})^{\hat{\otimes} n}\right\}$.

It is well known that $\mathcal{U}_{h}(\mathfrak{g})$ carries a (topological) ribbon Hopf algebra structure (see [5] or [10]). Then, $\mathcal{U}_{h}(\mathfrak{g})$ has an underlying (topological) quasi-triangular Hopf algebra structure with $R$-matrix $R$ (say). For the definition and properties of quasitriangular Hopf algebras and ribbon algebras, the reader may see [10]. It follows that there exists an invertible element $v \in \mathcal{U}_{h}(\mathfrak{g})$ such that

$$
S^{2}(x)=v x v^{-1} \quad \forall x \in \mathcal{U}_{h}(\mathfrak{g}) \quad \text { and } \quad v S(v) \text { is central }
$$

and a "ribbon element" $\theta$ which is central and satisfies (with notation as in [3]):

$$
\theta^{2}=v S(v) \quad \Delta(\theta)=\left(R_{21} R_{12}\right)^{-1}(\theta \otimes \theta) \quad \epsilon(\theta)=1 \quad S(\theta)=\theta .
$$

Following the proof of [3, Proposition 3], we can get a grouplike element $\sigma=\theta^{-1} v$ with

$$
\Delta(\sigma)=\sigma \otimes \sigma \quad \epsilon(\sigma)=1 \quad S(\sigma)=\sigma^{-1} .
$$

It follows from the proof of [3, Proposition 3] that $(\epsilon, \sigma)$ forms a modular pair in involution for the Hopf algebra $\mathcal{U}_{h}(\mathfrak{g})$.

Proposition 1.7. Let $\mathfrak{g}$ be a finite dimensional, complex, simple Lie algebra having quantized universal enveloping algebra $\mathcal{U}_{h}(\mathfrak{g})$ with generators $\left\{H_{i}, X_{ \pm i}\right\}, i=$ $1,2, \ldots, N$, as above. For each $i$, let $D_{i}$ denote the linear map on $\mathcal{U}_{h}(\mathfrak{g})$ given by the inner derivation $D_{i}=\left[H_{i},{ }_{--}\right]$on $\mathcal{H}$. Then, $D_{i} \in \operatorname{Bider}_{0}\left(\mathcal{U}_{h}(\mathfrak{g})\right)$, and hence there exists a corresponding linear operator $L_{i}: H C_{(\epsilon, \sigma)}^{*}\left(\mathcal{U}_{h}(\mathfrak{g})\right) \longrightarrow H C_{(\epsilon, \sigma)}^{*}\left(\mathcal{U}_{h}(\mathfrak{g})\right)$.

Proof. Choose any element $k \in \mathcal{U}_{h}(\mathfrak{g})$; then, we have

$$
\begin{aligned}
\Delta\left(D_{i}(k)\right)= & \Delta\left(H_{i} k-k H_{i}\right)=\Delta\left(H_{i}\right) \cdot \Delta(k)-\Delta(k) \Delta\left(H_{i}\right) \\
= & -\sum\left(H_{i} k_{(1)} \otimes k_{(2)}+k_{(1)} \otimes H_{i} k_{(2)}\right) \\
& -\sum\left(k_{(1)} H_{i} \otimes k_{(2)}+k_{(1)} \otimes k_{(2)} H_{i}\right) \\
& =\sum\left[H_{i}, k_{(1)}\right] \otimes k_{(2)}+\sum k_{(1)} \otimes\left[H_{i}, k_{(2)}\right] \\
= & \sum D_{i}\left(k_{(1)}\right) \otimes k_{(2)}+\sum k_{(1)} \otimes D_{i}\left(k_{(2)}\right)
\end{aligned}
$$

and hence $D_{i}$ is a coderivation. Since $\epsilon: \mathcal{U}_{h}(\mathfrak{g}) \longrightarrow \mathbb{C}$ is an $\mathbb{C}$-algebra morphism to the field $\mathbb{C}, \epsilon \circ D_{i}(k)=\epsilon\left(\left[H_{i}, k\right]\right)=0$. Also, $\operatorname{Im}(u)$ lies in the centre of $\mathcal{U}_{h}(\mathfrak{g})$ by definition, and hence $D_{i} \circ u=0$. Moreover,

$$
\begin{aligned}
D_{i} S(k)=H_{i} S(k)-S(k) H_{i} & =-S\left(H_{i}\right) S(k)+S(k) S\left(H_{i}\right) \\
& =-S\left(k H_{i}\right)+S\left(H_{i} k\right)=S\left(D_{i}(k)\right),
\end{aligned}
$$

where we recall that $S$ is an antihomomorphism and $S\left(H_{i}\right)=-H_{i}$. Also, we have $\delta \circ D_{i}(k)=\delta\left(H_{i} k-k H_{i}\right)=0$.

In order to prove that $D_{i} \in \operatorname{Bider}_{0}\left(\mathcal{U}_{h}(\mathfrak{g})\right)$, it remains to show that $D_{i}(\sigma)=0$. By definition, $\sigma=\theta^{-1} v$. Since $\mathcal{U}_{h}(\mathfrak{g})$ is quasi-triangular, it follows that $S^{2}\left(H_{i}\right)=$ $v H_{i} v^{-1}$. On the other hand, we know that $S\left(H_{i}\right)=-H_{i}$ and hence $S^{2}\left(H_{i}\right)=H_{i}$. Hence, $H_{i}$ commutes with $v$. Also, the ribbon element $\theta$ is central, and hence $\sigma=\theta^{-1} v$ commutes with $H_{i}$. It follows that $D_{i}(\sigma)=0$.

It now follows, exactly as in the proofs of Lemmas 1.2 and 1.3, that the linear map $D_{i}$ commutes with the face maps, degeneracies and cyclic operators of the cocyclic module $\mathcal{U}_{h}(\mathfrak{g})_{(\epsilon, \sigma)}^{\sharp, t o p}$. This induces a map

$$
L_{i}:=L_{D_{i}}: H C_{(\epsilon, \sigma)}^{*}\left(\mathcal{U}_{h}(\mathfrak{g})\right) \longrightarrow H C_{(\epsilon, \sigma)}^{*}\left(\mathcal{U}_{h}(\mathfrak{g})\right)
$$

just as in the proof of Proposition 1.4. 


\section{2. $\mathcal{H}$-Module struCture on $H C_{(\delta, \sigma)}^{*}(\mathcal{H})$}

In this section, we shall assume that our Hopf algebra $\mathcal{H}$ is the universal enveloping algebra $\mathcal{U}(\mathfrak{g})$ of a Lie algebra $\mathfrak{g}$. Then, from [11, Proposition 11.0.9] and [11, Theorem 13.0.1] (see also [1, 9]), this is equivalent to assuming that $\mathcal{H}$ is an irreducible, cocommutative Hopf algebra over $\mathbb{C}$ (for definitions, we refer the reader to [11]). Then, $\mathcal{H}=\mathcal{U}(\operatorname{Prim}(\mathcal{H}))$, where $\operatorname{Prim}(\mathcal{H})$ is the Lie algebra of primitive elements of $\mathcal{H}$ and $\mathcal{U}$ is the universal enveloping algebra functor. We shall show that the Hopf cyclic cohomology $H C_{\delta}^{*}(\mathcal{H})$ can be given the structure of a left $\mathcal{H}$-module.

Lemma 2.1. Let $\mathcal{H}$ be an irreducible, cocommutative Hopf algebra over $\mathbb{C}$ with a character $\delta: \mathcal{H} \longrightarrow \mathbb{C}$ such that $(\delta, 1)$ is a modular pair in involution. Let $h \in \mathcal{H}$ be a primitive element. Then, the inner derivation $D_{(h)}=\left[h,,_{-}\right]: \mathcal{H} \longrightarrow \mathcal{H}$ is a biderivation on $\mathcal{H}$. Also, $D_{(h)} \in \operatorname{Bider}_{0}(\mathcal{H})$, i.e.

$$
\epsilon \circ D_{(h)}=0 \quad D_{(h)} \circ u=0 \quad S D_{(h)}=D_{(h)} S \quad \delta \circ D_{(h)}=0 .
$$

Proof. Choose any element $k \in \mathcal{H}$; then, $h$ being primitive, we have

$$
\begin{aligned}
\Delta\left(D_{(h)}(k)\right) & =\Delta(h k-k h)=\Delta(h) \cdot \Delta(k)-\Delta(k) \Delta(h) \\
& =\sum\left(h k_{(1)} \otimes k_{(2)}+k_{(1)} \otimes h k_{(2)}\right)-\sum\left(k_{(1)} h \otimes k_{(2)}+k_{(1)} \otimes k_{(2)} h\right) \\
& =\sum\left[h, k_{(1)} \otimes k_{(2)}+\sum k_{(1)} \otimes\left[h, k_{(2)}\right]\right. \\
& =\sum D_{(h)}\left(k_{(1)}\right) \otimes k_{(2)}+\sum k_{(1)} \otimes D_{(h)}\left(k_{(2)}\right)
\end{aligned}
$$

and hence $D_{(h)}$ is a coderivation. Since $\epsilon: \mathcal{H} \longrightarrow \mathbb{C}$ is an $\mathbb{C}$-algebra morphism to the field $\mathbb{C}, \epsilon \circ D_{(h)}(k)=\epsilon([h, k])=0$. Also, $\operatorname{Im}(u)$ lies in the centre of $\mathcal{H}$ by definition, and hence $D_{(h)} \circ u=0$. Moreover,

$$
\begin{aligned}
D_{(h)} S(k)=h S(k)-S(k) h & =-S(h) S(k)+S(k) S(h) \\
& =-S(k h)+S(h k)=S\left(D_{(h)}(k)\right),
\end{aligned}
$$

where we recall that $S$ is an antihomomorphism and $S(h)=-h$ since $h$ is a primitive element of the Hopf algebra $\mathcal{H}$, which, in this case, happens to be the universal enveloping algebra of a Lie algebra. Finally, $\delta \circ D_{(h)}(k)=\delta(h k-k h)=0$.

Definition 2.2. Let $\mathcal{H}$ be a Hopf algebra over $\mathbb{C}$. If, for some $h \in \mathcal{H}$, the linear map induced by the commutator $D_{(h)}:=\left[h,,_{-}\right]: \mathcal{H} \longrightarrow \mathcal{H}$ is a biderivation, we say that $D_{(h)}$ is an inner biderivation.

We now have:

Proposition 2.3. Let $\mathcal{H}$ be an irreducible, cocommutative Hopf algebra over $\mathbb{C}$ with a character $\delta: \mathcal{H} \longrightarrow \mathbb{C}$ such that $(\delta, 1)$ is a modular pair in involution. Then the Hopf cyclic cohomology $H C_{\delta}^{*}(\mathcal{H})$ carries the structure of a left $\mathcal{H}$-module.

Proof. From Lemma 2.1 it follows that for each primitive element $h \in \operatorname{Prim}(\mathcal{H})$, we have a biderivation $D_{(h)} \in \operatorname{Bider}_{0}(\mathcal{H})$. From Proposition 1.4 it now follows that we have morphisms

$$
L_{(h)}:=L_{D_{(h)}}: H C_{\delta}^{*}(\mathcal{H}) \longrightarrow H C_{\delta}^{*}(\mathcal{H}) \quad \forall h \in \operatorname{Prim}(\mathcal{H})
$$

which, furthermore, by Proposition 1.6 satisfy $L_{\left[h, h^{\prime}\right]}=\left[L_{(h)}, L_{\left(h^{\prime}\right)}\right]$ for any two primitive elements $h, h^{\prime} \in \operatorname{Prim}(\mathcal{H})$. Therefore, the Lie algebra action of $\operatorname{Prim}(\mathcal{H})$ on $H C_{\delta}^{*}(\mathcal{H})$ by operators $L_{(h)}$ as in (2.4) endows $H C_{\delta}^{*}(\mathcal{H})$ with the structure of a left $\mathcal{H} \simeq \mathcal{U}(\operatorname{Prim}(\mathcal{H}))$-module. 


\section{The Hopf algebra $\mathcal{H}[D]$}

As in Section 2, we suppose throughout that $\mathcal{H}$ is a Hopf algebra over $\mathbb{C}$, equipped with a character $\delta: \mathcal{H} \longrightarrow \mathbb{C}$ such that $(\delta, 1)$ is a modular pair in involution. Further, we continue to assume that $\mathcal{H}$ is irreducible and cocommutative; i.e. it is the universal enveloping algebra of its primitive elements. Let $D$ be a biderivation on $\mathcal{H}$ which additionally satisfies condition $(\mathrm{c})$ of Section 1 , i.e. $D \in \operatorname{Bider}_{0}(\mathcal{H})$.

Now define $\mathfrak{L}=\operatorname{Prim}(\mathcal{H}) \oplus \mathbb{C} \cdot D$, i.e. the vector space obtained by formally adjoining $D$ to $\operatorname{Prim}(\mathcal{H})$. $\operatorname{Prim}(\mathcal{H})$ is already a Lie algebra, with a bracket [-_, _-_]. It is also clear that, $D$ being a coderivation, $D(\operatorname{Prim}(\mathcal{H})) \subseteq \operatorname{Prim}(\mathcal{H})$. We will now extend the Lie bracket on $\operatorname{Prim}(\mathcal{H})$ to all of $\mathfrak{L}$.

Lemma 3.1. On the vector space $\mathfrak{L}=\operatorname{Prim}(\mathcal{H}) \oplus \mathbb{C} \cdot D$, define the bracket $\left[\ldots,-{ }_{-}\right]_{D}$ by setting

$$
\begin{array}{ll}
{\left[h_{1}, h_{2}\right]_{D}=-\left[h_{2}, h_{1}\right]_{D}=\left[h_{1}, h_{2}\right]} & \text { if } h_{1}, h_{2} \in \operatorname{Prim}(\mathcal{H}) \\
{[D, h]_{D}=-[h, D]_{D}=D(h)} & \text { if } h \in \operatorname{Prim}(\mathcal{H}) \\
{[D, x D]_{D}=[x D, D]_{D}=0} & \text { if } x \in \mathbb{C}
\end{array}
$$

and extending by bilinearity to a bracket on all of $\mathfrak{L} \times \mathfrak{L}$. Then, [--, --] $]_{D}: \mathfrak{L} \times \mathfrak{L} \longrightarrow \mathfrak{L}$ defines a Lie algebra structure on $\mathfrak{L}$.

Proof. We need to check that, given $x, y, z \in \mathfrak{L},\left[x,[y, z]_{D}\right]_{D}+\left[y,[z, x]_{D}\right]_{D}+$ $\left[z,[x, y]_{D}\right]_{D}=0$. It suffices to check that, given $y, z \in \operatorname{Prim}(\mathcal{H}),\left[D,[y, z]_{D}\right]_{D}+$ $\left[y,[z, D]_{D}\right]_{D}+\left[z,[D, y]_{D}\right]_{D}=0$. We have

$$
\begin{aligned}
& {\left[D,[y, z]_{D}\right]_{D}=D(y z-z y)=D(y) z+y D(z)-D(z) y-z D(y)} \\
& {\left[y,[z, D]_{D}\right]_{D}=-[y, D(z)]_{D}=-y D(z)+D(z) y} \\
& {\left[z,[D, y]_{D}\right]_{D}=[z, D(y)]_{D}=z D(y)-D(y) z} \\
& \Rightarrow\left[D,[y, z]_{D}\right]_{D}+\left[y,[z, D]_{D}\right]_{D}+\left[z,[D, y]_{D}\right]_{D}=0 .
\end{aligned}
$$

Let us, therefore, denote by $\mathcal{H}[D]$ the universal enveloping algebra $\mathcal{U}(\mathfrak{L})$. Then $\mathcal{H}$ embeds into $\mathcal{H}[D]$, and it follows from [11, Proposition 11.0.9] that $\mathcal{H}[D]$ is an irreducible, cocommutative Hopf algebra. Extend $\delta: \mathcal{H} \longrightarrow \mathbb{C}$ to $\delta_{D}: \mathcal{H}[D] \longrightarrow \mathbb{C}$ by setting $\delta_{D}(h)=\delta(h)$ for $h \in \operatorname{Prim}(\mathcal{H})$ and $\delta_{D}(D)=0$. Then, there exists a morphism of Hopf cyclic cohomologies

$$
i: H C_{\delta}^{*}(\mathcal{H}) \longrightarrow H C_{\delta_{D}}^{*}(\mathcal{H}[D]) .
$$

Also, $D$ is a primitive element of the Hopf algebra $\mathcal{H}[D]$. The following proposition now shows that the operator $L_{D}: H C_{\delta}^{*}(\mathcal{H}) \longrightarrow H C_{\delta}^{*}(\mathcal{H})$ can be described as an operator induced by an inner biderivation.

Proposition 3.2. The inner biderivation $\left[D,{ }_{-}\right]_{D}$ of $\mathcal{H}[D]$ corresponding to $D$ induces an endomorphism of $H C_{\delta_{D}}^{*}(\mathcal{H}[D])$, which we denote by $L_{(D)}$. Then, we have a commutative diagram

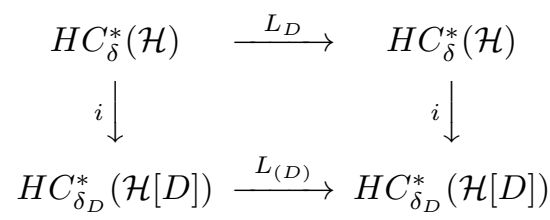


Proof. Following Proposition 2.3 , the inner biderivation $[D, \ldots]]_{D}$ of $\mathcal{H}[D]$ induces an endomorphism $L_{(D)}: H C_{\delta}^{*}(\mathcal{H}[D]) \longrightarrow H C_{\delta}^{*}(\mathcal{H}[D])$. Since $\mathcal{H} \simeq \mathcal{U}(\operatorname{Prim}(\mathcal{H}))$, it follows that $D: \mathcal{H} \longrightarrow \mathcal{H}$ is completely determined by its action on $\operatorname{Prim}(\mathcal{H})$. Hence, for any $h \in \mathcal{H}$ :

$$
i(D(h))=[D, i(h)]_{D} \in \mathcal{H}[D] .
$$

The commutativity of (3.4) follows easily from this.

\section{ACKNOWLEDGEMENT}

The author is grateful to the referee for important suggestions and comments. In particular, it was suggested by the referee that a "quantum example" of a map $L_{D}$ induced by a biderivation $D$ be constructed and included in the paper.

\section{REFERENCES}

[1] Cartier, P.: Hyperalgèbres et groupes de Lie formels. Séminaire Sophus Lie, 2e année 1955/56, Faculté des Sciences de Paris.

[2] Connes, Alain; Moscovici, Henri: Cyclic cohomology and Hopf algebra symmetry. Conference Moshé Flato 1999 (Dijon). Lett. Math. Phys., 52 (2000), no. 1, 1-28. MR.1800488 (2002d:58009)

[3] Connes, Alain; Moscovici, Henri: Cyclic cohomology and Hopf algebras. Lett. Math. Phys., 48 (1999), no. 1, 97-108. MR1718047(2000j:16061)

[4] Connes, A.; Moscovici, H.: Hopf algebras, cyclic cohomology and the transverse index theorem. Comm. Math. Phys., 198 (1998), no. 1, 199-246. MR.1657389 (99m:58186)

[5] Drinfel'd, V.: Quantum Groups, Proc. Internat. Congress Mathematicians (Berkeley, 1986), Vol. 1, Amer. Math. Soc., Providence, RI, 1987, 798-820. MR934283 (89f:17017)

[6] Hajac, Piotr M.; Khalkhali, Masoud; Rangipour, Bahram; Sommerhäuser, Yorck: Hopf-cyclic homology and cohomology with coefficients. C. R. Math. Acad. Sci. Paris, 338 (2004), no. 9, 667-672. MR2065371 (2005b:19002)

[7] Hajac, Piotr M.; Khalkhali, Masoud; Rangipour, Bahram; Sommerhäuser, Yorck: Stable anti-Yetter-Drinfeld modules. C. R. Math. Acad. Sci. Paris, 338 (2004), no. 8, 587-590. MR2056464 (2005a:16056)

[8] Loday, Jean-Louis: Cyclic Homology, Grundlehren der Mathematischen Wissenschaften, 301, Springer-Verlag, Berlin, 1998. MR,1600246 (98h:16014)

[9] Milnor, John W.; Moore, John C.: On the structure of Hopf algebras. Ann. of Math. (2), 81 (1965), 211-264. MR0174052 (30:4259)

[10] Reshetikhin, N. Yu.; Turaev, V. G.: Ribbon graphs and their invariants derived from quantum groups. Comm. Math. Phys., 127 (1990), 1-26. MR1036112 (91c:57016)

[11] Sweedler, M. E.: Hopf Algebras, Mathematics Lecture Note Series, 44, W. A. Benjamin, Inc., New York, 1969. MR0252485(40:5705)

Department of Mathematics, Johns Hopkins University, 3400 N. Charles Street, BalTIMORE, MARYLAND 21218

E-mail address: abanerje@math.jhu.edu

Current address: Department of Mathematics, Ohio State University, 231 W. 18th Avenue, Columbus, Ohio 43210 